\title{
Primeiro registro de Thaumastocoris peregrinus para o estado de Santa Catarina, e novas áreas de ocorrência para o Rio Grande do Sul, Brasil
}

\author{
First record of Thaumastocoris peregrinus for the state of Santa Catarina, and new areas of \\ occurrence for the Rio Grande do Sul, Brazil
}

\author{
Marcoandre Savaris $^{\mathrm{I}^{*}}$ Silvana Lampert ${ }^{\mathrm{I}}$ Paulo Roberto Valle da Silva Pereira $^{\mathrm{I}}$ \\ José Roberto Salvadori" ${ }^{\mathrm{II}}$
}

- NOTA -

\section{RESUMO}

Thaumastocoris peregrinus é relatado pela primeira vez para o estado de Santa Catarina atacando plantas isoladas de Eucalyptus camaldulensis, Eucalyptus tereticornis e reflorestamentos de Eucaliptus grandis localizados no interior dos municípios de Chapecó, Cordilheira Alta, Xaxim e Xanxerê. No Rio Grande do sul, esse inseto já é encontrado em 10 localidades do sul do estado, e são registradas novas áreas de ocorrência nos municípios de Ernestina, Passo Fundo, Marau, Coxilha, Getúlio Vargas, Erechim, São Valentim, Erval Grande, Nonoai, Três Palmeiras, Ronda Alta, Pontão e Quatro Irmãos.

Palavras-chave: Eucalyptus, percevejo-bronzeador, praga florestal.

\begin{abstract}
Thaumastocoris peregrinus is recorded for the first time in the state of Santa Catarina attacking isolated plants of Eucalyptus camaldulensis, Eucalyptus tereticornis and reforestation of Eucalyptus grandis located within the municipalities of Chapecó, Cordillera Alta, Xaxim and Xanxerê. In Rio Grande do Sul this insect is already found in 10 locations, and new areas of occurrence are recorded in the municipalities of Ernestina, Passo Fundo, Marau, Coxilha, Getúlio Vargas, Erechim, São Valentim, Erval Grande, Nonoai, Três Palmeiras, Ronda Alta, Pontão and Quatro Irmãos.
\end{abstract}

Key words: Eucalyptus, bronze bug, forestry pest.

Thaumastocoris peregrinus Carpintero e Dellapé, 2006 (Hemiptera, Thaumastocoridae), é uma espécie nova e o único membro da subfamília
Thaumastocorinae com ocorrência registrada nas Américas. Originalmente, as cinco espécies deste gênero são encontradas na Austrália (T. australicus, T. hackeri, T. petilus e T. macqueeni) e África do Sul (T. peregrinus) (CARPINTERO\& DELLAPÉ, 2006). Nas Américas, sua ocorrência foi registrada pela primeira vez na Argentina por NOACK \& COVIELLA (2006), erroneamente identificado como $\boldsymbol{T}$. australicus.

No Brasil, a ocorrência desse inseto foi relatada pela primeira vez em maio de 2008 , no município de São Francisco de Assis, interior do estado do Rio Grande do Sul, sobre clone híbrido de Eucaliptus grandis x Eucaliptus urophylla, conhecido como urograndis (WILCKEN et al., 2010; BARBOSA et al., 2010). Até o momento, T. peregrinus foi encontrado em mais de 40 municípios no estado de São Paulo, além da ocorrência nos estados do Rio de Janeiro, Minas Gerais, Mato Grosso do Sul e Paraná, sempre tendo espécies de Eucalyptus como hospedeiros (WILCKEN, 2008; BARBOSA et al., 2010; WILCKEN et al., 2010).

O percevejo-bronzeador-do-eucalipto $\boldsymbol{T}$. peregrinus é um inseto fitófago com hábito alimentar sugador. Seus ovos têm coloração preta e são colocados em agrupamentos sobre as folhas, em quantidade variável. As ninfas apresentam coloração castanho-clara, com a presença de mancha escura na região dorsal do abdome. Os adultos possuem corpo achatado de coloração geral castanho-amarelada, comprimento médio de três milímetros e se caracterizam

IEmbrapa Trigo, BR 285, km 294, CP 451, 99001-970, Passo Fundo, RS, Brasil. E-mail. masavaris@yahoo.com.br. Autor para correspondência.

"Faculdade de Agronomia e Medicina Veterinária, Universidade de Passo Fundo (UPF), Passo Fundo, RS, Brasil. 
por apresentarem placas mandibulares desenvolvidas, ausência de pulvilo, apêndices apicais em todas as tíbias e genitália masculina assimétrica (JACOBES \& NESSER, 2005; CARPINTERO \& DELLAPÉ, 2006; BUTTON, 2010). Segundo CASSIS et al. (1999), os Thaumastocorinae utilizam como hospedeiros diversas dicotiledôneas, incluindo espécies de Eucalyptus (Myrtaceae), Banksia (Protaceae), Acacia (Fabaceae) e Elaeocarpus (Elaeocarpaceae).

O gênero Thaumastocoris é considerado praga de espécies de Eucalyptus (JACOBES \& NESSER, 2005; WILCKEN, 2008; WILCKEN et al., 2010). Dentre os principais danos que esta praga causa às plantas de eucalipto, destacam-se o bronzeamento e a redução da capacidade fotossintética da planta, culminando com o secamento e a queda das folhas, com consequente prejuízo ao seu desenvolvimento (JACOBS \& NESSER, 2005). Em casos de densidades populacionais altas, em espécies de eucaliptos suscetíveis à praga, os danos causados podem resultar na morte das plantas (WILCKEN, 2008; WILCKEN et al., 2010).

Dessa forma, o objetivo deste trabalho é relatar a ocorrência de $\boldsymbol{T}$. peregrinus atacando plantas de Eucalyptus no estado de Santa Catarina e ampliar a distribuição da espécie no Rio Grande do Sul.

As observações de $\boldsymbol{T}$. peregrinus foram realizadas no período de março a outubro de 2009, nos municípios de Chapecó, Cordilheira Alta, Xaxim e Xanxerê, região oeste do estado de Santa Catarina e, nos municípios de Ernestina, Passo Fundo, Marau, Coxilha, Getúlio Vargas, Erechim, São Valentim, Erval Grande, Nonoai, Três Palmeiras, Ronda Alta, Pontão e Quatro Irmãos, região do planalto médio e norte do estado do Rio Grande do Sul (Figura 1). Foram observados aleatoriamente ramos e folhas em árvores isoladas e povoamentos de diferentes espécies de Eucalyptus, existentes nas margens de estradas e rodovias. Ao ser constatada a presença da espéciepraga, exemplares foram coletados, fixados em álcool $70 \%$ e analisados no laboratório de Entomologia da Embrapa Trigo em Passo Fundo, Rio Grande do Sul, a fim de confirmar a identificação taxonômica e observar as diferentes fases de desenvolvimento (ovo, ninfa e adulto). As áreas de ocorrência foram georreferenciadas e os sintomas associados ao dano de $\boldsymbol{T}$. peregrinus nas plantas atacadas foram caracterizados.

T. peregrinus foi encontrado inicialmente em abril de 2009 no município de Chapecó em brotações, com aproximadamente três metros de altura, e plantas adultas de $\boldsymbol{E}$. camaldulensis. Nesse mesmo município, também foi observada a infestação do inseto em uma área de reflorestamento de $\boldsymbol{E}$. grandis. No município de Cordilheira Alta, T. peregrinus foi encontrado em plantas de $\boldsymbol{E}$. grandis, no município de Xaxim em plantas isoladas de $\boldsymbol{E}$. camaldulensis próximas à rodovia BR 282 e, no município de Xanxerê, este inseto foi detectado em árvores das espécies $\boldsymbol{E}$. camaldulensis e $\boldsymbol{E}$. tereticornis. Este é o primeiro registro de $\boldsymbol{T}$. peregrinus infestando plantas de

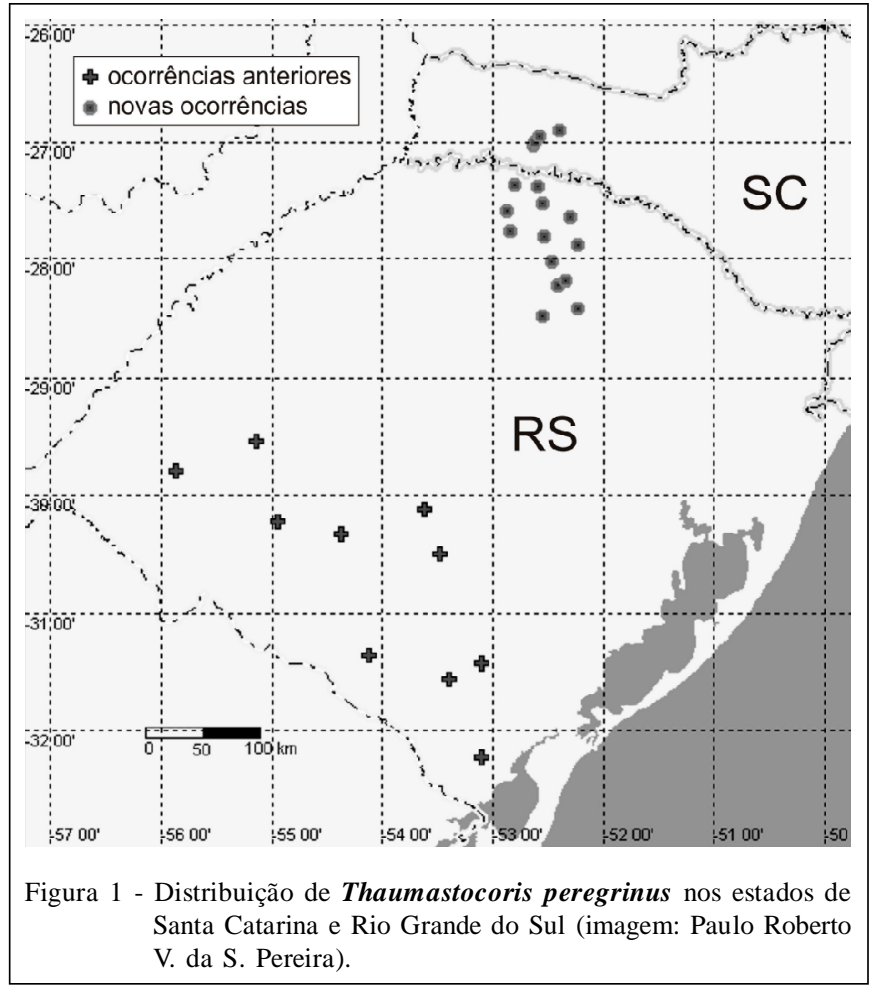

Ciência Rural, v.41, n.11, nov, 2011. 
diferentes espécies de Eucalyptus para o estado de Santa Catarina.

No Rio Grande do Sul, essa praga já é encontrada em 10 localidades do sul do estado e com este trabalho são registradas novas áreas de ocorrência nos municípios de Ernestina, Passo Fundo, Marau, Coxilha, Getúlio Vargas, Erechim, São Valentim, Erval Grande, Nonoai, Três Palmeiras, Ronda Alta, Pontão e Quatro Irmãos. Nos locais onde este inseto foi detectado, foram observados concomitantemente ovos, ninfas e adultos (Figura 2).

Em todas as situações de presença de $\boldsymbol{T}$. peregrinus, foi evidente o sintoma de bronzeamento e, nos casos mais severos, a queda intensa de folhas das plantas de eucalipto (Figura 3a). Os mesmos sintomas foram observados por JACOBS \& NESSER (2005) e NOACK \& COVIELLA (2006) em infestações na África do Sul e na Argentina, respectivamente, sendo observada neste levantamento grande concentração de adultos, ninfas e posturas dividindo o mesmo ambiente nas folhas das plantas atacadas (Figura $3 b$ ). Embora a infestação em diferentes alturas das plantas atacadas não tenha sido avaliada, a presença do sintoma de bronzeamento e queda de folhas em toda a árvore é um indicativo de que os insetos estão distribuídos amplamente no hospedeiro. JACOBS \& NESSER (2005) corroboram esta hipótese ao sugerirem que as infestações são maiores na copa das árvores.

Os resultados obtidos neste trabalho mostram que o inseto $T$. peregrinus apresenta alto potencial de dispersão e que também está adaptado à região sul do Brasil. Dessa maneira, essas novas ocorrências evidenciam a necessidade de maiores estudos sobre bioecologia e manejo desse inseto, além de, ao mesmo tempo, servirem de alerta para a ameaça que T. peregrinus representa para os plantios de eucalipto.

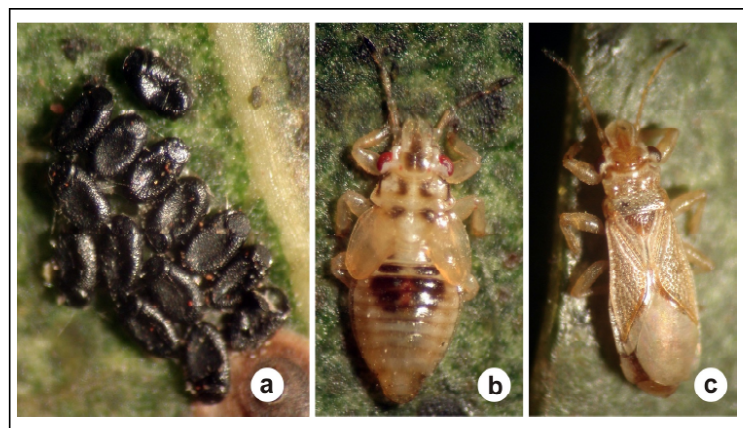

Figura 2 - Thaumastocoris peregrinus. a) postura; b) ninfa e c) adulto (imagens: Paulo Roberto V. da S. Pereira).

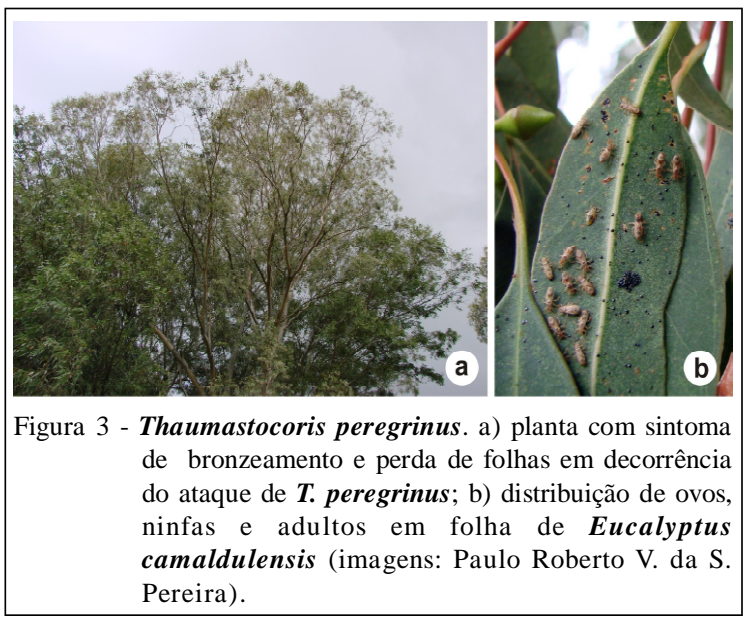

\section{REFERÊNCIAS}

BARBOSA, L.R. et al. Registro de Thaumastocoris peregrinus (Hemiptera, Thaumastocoridae) no Estado do Paraná. Pesquisa Florestal Brasileira, v.30, p.75-77, 2010. Disponível em: < h t t p : / / w w w . c n p f.e m b r a a . b r / i mage n s / Nota_Cientifica_Percevejo_bronzeado.pdf $>$. Acesso em: 11 ago. 2009. doi: $10.4336 / 2010 . p f b .30 .61 .75$.

BUTTON, G. Thaumastocoris peregrinus. Forestry facts, 2007. 2p. Disponível em: <http://www.nctforest.com/ show page. asp $? \mathrm{id}=44 \&$ contentid $=423 \&$ catid $=24>$. Online. Acesso em: 01 jul.2010.

CARPINTERO, D.L.; DELlAPÉ, P.M. A new species of Thaumastocoris Kirkaldy from Argentina (Heteroptera: Thaumastocoridae: Thaumastocorinae). Zootaxa, v.1228, p.61-68, 2006. Disponível em: <http://www.mapress.com/ zootaxa/2006f/z01228p068f.pdf>. Acesso em: 28 jul. 2010.

CASSIS, G. et al. A review of Onymocoris (Heteroptera: Thaumastocoridae), with a new species, and notes on hosts and distributions of other thaumastocorid species. Acta Societatis Zoologische Bohemoslavaca, v.63, p.19-36, 1999.

JACOBS, D.H.; NESSER, S. Thaumastocoris australicus Kirkaldy (Hetereptera: Thaumastocoridae): a new insect arrival in Sounth África, damaging to Eucalyptus trees. South African Journal of Science, v.101, p.233-236, 2005.

LIN, N.Q. et al. The Australian genera of Mymaridae (Hymenoptera: Chalcidoidea). Zootaxa, v.1596, 111p, 2007. Disponível em: <http://www.mapress.com/zootaxa/2007f/ z01596p111f.pdf>. Acesso em: 10 jun. 2010.

NOACK, A.E.; COVIELLA, C.E. Thaumastocoris australicus Kirkaldy (Hemiptera: Thaumastocoridae): first record of this invasive pest of Eucalyptus in the Americas. General and Applied Entomology, v.35, p.13-14, 2006.

WILCKEN, C.F. Percevejo bronzeado do eucalipto Thaumastocoris peregrinus (Hemiptera: Thaumastocoridae): ameaça às florestas de eucalipto brasileiras. IPEF Programa de proteção florestal - PROTEF/IPEF. Botucatu, SP, Setembro de 2008. Disponível em: <http://www.ipef.br/ protecao/alerta-percevejo.pdf $>$. Oline. Acesso em: 05 jul. 2009.

WILCKEN, C.F. et al. Bronze bug Thaumastocoris peregrinus Carpintero and Dellape (Hemiptera: Thaumastocoridae) on Eucalyptus in Brazil and its distribution. Journal of Plant Protection research, v.50, n.2, p.201-205, 2010. Disponível em: <http:// www.plantprotection.pl/PDF/50(2)/JPPR_50(2)_14_Wilcken.pdf >. Acesso em: 10 nov. 2010. 